\title{
Transposition
}

Musique et Sciences Sociales

$4 \mid 2014$

Musique et conflits armés après 1945

\section{De la musique à ses objets et ses images.}

Entretien avec Florence Gétreau

From music to its objects and images. Interview with Florence Gétreau

\section{Fanny Gribenski et Isabelle Mayaud}

\section{(2) OpenEdition}

Journals

Édition électronique

URL : http://journals.openedition.org/transposition/980

DOI : $10.4000 /$ transposition.980

ISSN : 2110-6134

Éditeur

CRAL - Centre de recherche sur les arts et le langage

Référence électronique

Fanny Gribenski et Isabelle Mayaud, "De la musique à ses objets et ses images. », Transposition [En ligne], 4 | 2014, mis en ligne le 15 juillet 2014, consulté le 14 novembre 2019. URL : http://

journals.openedition.org/transposition/980; DOI : 10.4000/transposition.980

Ce document a été généré automatiquement le 14 novembre 2019.

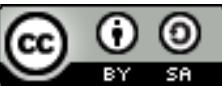

La revue Transposition est mise à disposition selon les termes de la Licence Creative Commons Attribution - Partage dans les Mêmes Conditions 4.0 International. 


\title{
De la musique à ses objets et ses images.
}

\author{
Entretien avec Florence Gétreau \\ From music to its objects and images. Interview with Florence Gétreau
}

\author{
Fanny Gribenski et Isabelle Mayaud
}

\section{NOTE DE L'ÉDITEUR}

Réalisé par Fanny Gribenski et Isabelle Mayaud, le 17 juin 2013.

\section{Entretien}

FANNY GRIBENSKI ET ISABELLE MAYAUD - Comment expliquez-vous votre double carrière de conservatrice et de chercheuse?

Florence Gétreau - Mes années de formation ont été déterminantes dans l'affirmation d'une double vocation de chercheuse et de conservatrice de musée. J'ai fait un cursus de lettres et d'histoire de l'art à la faculté d'Aix en Provence et j'ai alors travaillé sur la chanson de Roland, à la croisée de ces deux disciplines. Puis, je suis venue à Paris, mon idée étant de m'inscrire à la formation de préparation au concours des musées nationaux. J'avais vraiment une vocation forte pour les musées. Jacques Thuillier ${ }^{1}$, alors responsable du département d'histoire de l'art moderne à la Sorbonne m'a conseillé de commencer par faire un catalogue raisonné de musée dans le cadre d'une maîtrise. Mon mémoire fut consacré aux Peintures et dessins français du XVIII siècle du musée Jacquemart-André de Paris. J'en ai publié une version profondément remaniée l'an dernier grâce à l'Institut de France et aux éditions Michel de Maule. C'est aussi à cette époque que j'ai suivi en Sorbonne le séminaire de muséologie générale de Georges-Henri Rivière ${ }^{2}$. Rivière faisait deux types de cours : des cours théoriques et, tous les mercredis, des cours pratiques dans les institutions où nous étions reçus par les directeurs et les équipes. C'est comme cela qu'un jour, 
nous sommes allés au Musée du Conservatoire de Paris. Et là, j'ai eu l'une des très grandes émotions de ma vie: au cours de la visite, Hubert Bédard, claveciniste et responsable de l'atelier de restauration du musée, a joué un clavecin du XVIII ${ }^{e}$ siècle de Jean-Claude Goujon. L'audition de cet instrument a été une véritable révélation. Deux mois après, je rencontrais Madame de Chambure $^{3}$, troisième personne exceptionnelle qui a joué, comme Thuillier et Rivière, un rôle déterminant dans ma carrière.

Le musée du Conservatoire a longtemps été au centre de ma vie, non seulement professionnelle, mais aussi quotidienne. J'y ai d'abord fait un stage; puis un poste à mi-temps d'assistante s'est libéré. Sitôt ma maîtrise en poche, j'ai décidé de commencer une thèse sous la direction de Jacques Thuillier, sur l'histoire des collections du Musée instrumental du Conservatoire. Alors que j'étais conservateuradjoint à plein temps, j'ai poursuivi ma thèse soutenue finalement en 1991. J'ai contribué dès 1975 à l'équipe du CNRS créée auprès de ce musée par Madame de Chambure en 1967 et dirigée par Jacques Thuillier à partir de 1974. Elle était consacrée à l'étude interne et externe des instruments de musique et à l'iconographie musicale. Dès 1977, auprès de Georges Henri Rivière et Josiane Bran-Ricci, j'ai contribué aux premières réflexions sur l'établissement d'un musée de la Musique. Et de 1987 à 1992, j'ai été Chef de projet du musée de la musique auprès de l'Établissement public constructeur de La Villette. J'ai appris ce que c'est qu'un grand projet culturel de l'État, avec une dimension fortement politique.

Après avoir été en quelque sorte porteuse de ce musée de la musique, pour des raisons de politique culturelle, j'en ai été écartée, à deux ans de l'ouverture. Comme j'étais conservateur des musées nationaux, je suis allée voir où l'on m'accepterait, sachant que j'avais hérité en 1993 de la direction de l'équipe CNRS autrefois dirigée par Madame de Chambure, puis par J. Thuillier. Alors que je devenais conservateur responsable du département de la Musique et de la parole, mon équipe de recherche était aussi intégrée au Musée des Arts et Traditions Populaires, en tant que deuxième laboratoire CNRS. J'ai travaillé pour les deux équipes associées au musée, entre lesquelles j'ai joué un rôle d'intermédiaire. En 1995, j'ai formulé le vœu de faire une exposition sur les Musiciens des rues de Paris. ${ }^{4}$. C'était un projet d'anthropologie historique, ce qui n'était pas très fréquent à l'époque - chez les ethnologues, on travaillait alors plus volontiers sur les banlieues, et l'on ne cultivait pas trop ce qui avait une dimension historique. Cela a été un des très grands moments de ma carrière au MNATP, un moment de grande créativité. Nous avons constitué un groupe de réflexion avec des ethnomusicologues, des musiciens professionnels, des praticiens de la rue et des historiens. Au MNATP, j'ai tenté de faire fructifier des travaux de fond avec des objectifs très divers, aussi bien scientifiques que de restitution culturelle. En 2004, l'idée d'une relocalisation du MNATP à Marseille s'est précisée. Je n'arrivais pas à adhérer au projet, qui mélangeait trois choses très importantes mais difficiles à articuler : l'ethnologie de la France, de l'Europe, et de la Méditerranée.

Ayant contribué dès 1996 au nouvel Institut de recherche sur le patrimoine musical en France (UMR 200 du CNRS, soutenue par le Ministère de la Culture et la BnF, où je suis responsable du programme "Organologie et Iconographie musicale »), j'ai été chargée de la direction de ce laboratoire en 2004, la Direction des musées de France ayant accepté de me mettre à la disposition du CNRS pour quelques années. Mon ancienne équipe de recherche du MNATP avait été intégrée à ce laboratoire. Puis, en 
2005, j'ai décidé de passer le concours externe du CNRS. Depuis, je n'ai plus de collections sous ma responsabilité, ce qui me manque parfois.

FG ET IM - Comment ce parcours singulier s'est-il traduit en termes d'orientations scientifiques?

FG - En termes d'inscription disciplinaire, je me suis sentie toute ma vie en marge des sujets dominants, ce qui est parfois une force. Chaque discipline ou sous-discipline s'est construit une image de ses limites, des compétences, des méthodes et des procédures d'adoubement. Je me suis formée à plusieurs domaines avec finalement une prédilection pour l'organologie et l'iconographie, toutes deux des disciplines sœurs de la musicologie que j'ai réunies dans la revue Musique-Images-Instruments que j'ai fondée en 1995 avec l'aide du CNRS et du Ministère de la Culture (cf. supra). J'aimerais à l'avenir faire une sorte de vade-mecum, un manuel de ces deux sousdisciplines à partir de mon habilitation à diriger des recherches, laquelle a porté sur l'Histoire des instruments et les représentations de la musique en France.

La transmission et le partage des travaux occupent une place importante dans ma trajectoire professionnelle. Je dois cette approche de la réflexion en groupe au CNRS, dont c'est l'une des forces. Grâce à Yves Gérard aussi, que j'ai fréquenté pendant des années, et qui m'a demandé en 1994 de donner des cours aux étudiants du Conservatoire, j'ai pu partager mes recherches et les travaux que j'aime. Formuler une critique rétrospective sur ce qu'on a pensé trouver, c'est difficile. En fait, je pense que l'on a besoin du feedback des autres, de la relecture, de l'écho... Je trouve qu'il n'y a rien de pire dans nos milieux que les gens qui refusent que l'on corrige ou modifie leurs textes. J'ai beaucoup cru au collectif et j'ai toujours plusieurs chantiers communs en cours. Les années passant il y a aussi urgence à finaliser plusieurs recherches personnelles non achevées faute de temps. Au début de ma carrière et jusqu'à la fin de mon engagement au MNATP, j'ai fait beaucoup de service public. Depuis que je suis au CNRS, je n'ai pas de service public à proprement parler à assumer, mais j'ai tellement de charges pour la collectivité que ça revient au même.

Je suis également très impliquée à l'international et j'ai acquis une reconnaissance qui s'est traduite par un certain nombre de distinctions ces dix dernières années ${ }^{5}$. Je fais aussi partie de plusieurs sociétés savantes : The Academy of Europe et la Société internationale de musicologie notamment. Cela m'importe beaucoup d'être présente dans des réunions internationales, cela participe d'un besoin d'ouverture présent depuis le début de ma carrière.

FG ET IM - Quels sont vos projets actuels?

FG - Le $14^{\mathrm{e}}$ volume de la revue Musique $\bullet$ Images $•$ Instruments est sous presse, il portera sur un curieux instrument, le serpent $t^{6}$. Il y a aussi plusieurs ouvrages collectifs en cours, comme celui avec Jean Duron sur l'orchestre à cordes sous Louis $\mathrm{XIV}^{7}$, ou le volume collectif sur Henri Prunières ${ }^{8}$. Il y a encore le livre Voir la musique $e^{9}$, qui est sans doute celui qui me tient le plus à cœur.

FG ET IM - Dans votre actualité, il y a aussi une exposition sur la musique et la guerre à Péronne $^{10}$ : comment êtes-vous devenue commissaire d'exposition?

FG - Mon premier commissariat d'exposition date de 1980. Il s'agissait d'une exposition, dans le cadre de l'Année européenne de la musique, sur la facture instrumentale. J'avais voulu montrer que chaque pays avait eu une prééminence dans 
un domaine ou l'autre: j'ai proposé un tour d'Europe de la facture instrumentale. Chaque pays avait aussi sa manière de penser l'organologie ${ }^{11}$. En 1988, j'ai réalisé une autre exposition sur les Instrumentistes et luthiers parisiens pour la mairie de Paris ${ }^{12}$ et puis plusieurs autres encore, au MNATP notamment.

En 2007, il y a eu un beau colloque à Péronne ${ }^{13}$, pour lequel on m'avait demandé de faire un travail sur les instruments de soldats. J'ai accepté à condition de pouvoir faire un travail systématique sur les collections de l'Historial. C'est comme cela que j'ai découvert qu'il y avait là les carnets et les manuscrits de Gervais sur son fameux violoncelle de tranchée. La conservatrice de l'Historial de Péronne en charge aussi de l'abbaye de Saint-Riquier, m'a d'abord commandé une exposition sur la musique dans les tableaux des musées de province en 2009. Puis elle m'a confié le commissariat de celle de 2014 qui sera intitulée Entendre la guerre: sons, musiques et silence en 14-18. Dans le cadre de la préparation de cette exposition, je travaille avec un comité scientifique (historiens et musicologues) et une équipe de production qui est à Péronne.

FG ET IM - Comment s'est organisé le travail avec le comité scientifique?

FG - Le comité scientifique a défini les axes, les points forts, la structuration, l'intitulé ; le sommaire du catalogue reflète très bien le parcours théorique. La question, ensuite, c'est : quels objets expose-t-on ? Comment est-ce qu'on incarne les idées? Par exemple, sur la chanson, on doit opérer des sélections drastiques, car que retenir? A l'inverse, sur certains thèmes, les documents sont rares. Le travail de notre comité a permis d'opter non pas pour une exposition sur la musique pendant la Grande Guerre, mais pour un triptyque permettant de prendre en compte le son inouï de la guerre, sa violence, sa nouveauté, alors même que quasi aucun témoignage matériel ne nous a été conservé. Toute la partie portant sur la musique (au front, à l'arrière, dans les camps, dans les zones occupées) constitue ensuite une sorte de contre-bruit. Enfin il nous est apparu essentiel d'évoquer le silence : celui qui marque subitement l'armistice, mais aussi celui qui est redouté par les soldats saturés de violence sonore. Il est ainsi question de la dimension sonore, comme on le voit dans le titre. Mais comment faire passer des sensations ? C'est un sujet très dur, la guerre. Je ne m'étais jamais vraiment penchée sur la question jusqu'à cette exposition.

FG ET IM - Passionnante question, oui, comment faire passer des sensations ?

FG - Nous avons la chance d'avoir dans l'équipe de scénographie Luc Martinez qui est compositeur et qui a à son actif une longue expérience auprès des musées dédiés à la musique. Plusieurs thématiques de l'exposition feront l'objet de puits sonores où l'on pourra entendre des extraits de répertoires très divers (Musiques de défilés, chansons, œuvres de circonstance et de deuil etc.). Mais une salle particulière sera dédiée à une création sonore de Luc Martinez, intitulée «Inouï » qui permettra de prendre conscience, jusque dans ses aspects «vibratoires » de l'impact physique et sensible du son de la guerre. C'est un sujet nouveau pour une exposition, à la croisée de toutes sortes d'interrogations. Là encore, il y a une espèce de porosité du sujet, des domaines d'expertises des différents partenaires et collaborateurs, et c'est ce qui me fait avancer. 


\section{BIBLIOGRAPHIE}

\section{Bibliographie sélective}

\section{Ouvrages}

Musée Jacquemart-André. Peintures et dessins de l'école française. Catalogue raisonné, Paris, Institut de France : Michel de Maule, 2011, 413 p., 160 ill. coul., 50 ill. $n$ et b.

Voir la musique. Les sujets musicaux dans les œuvres d'art du XVI ${ }^{e}$ au XX $X^{e}$ siècle, Musée départemental de l'Abbaye de Saint-Riquier, 2009, 154 p., 76 ill.

Aux origines du Musée de la Musique : les collections instrumentales du Conservatoire de Paris. 1793-1993, Paris, Klincksieck / Réunion des Musées Nationaux, 1996, 800 p., 120 ill.

\section{Direction d'ouvrages}

Chopin e il suono di Pleyel - Chopin and the Pleyel sound - Chopin et le son Pleyel ; Arte e musica nella Parigi romantica - Art and Music in Romantic Paris - Art et musique dans le Paris romantique, Milan, Villa Medici Giulini, 2010, 380 p., 190 ill.

Musique, esthétique et société en France au XIX siècle, avec Damien Colas et Malou Haine, Liège, Mardaga, 2007, 336 p.

Direction de la revue Musique •Images • Instruments. Revue française d'organologie et d'iconographie musicale. Derniers volumes thématiques parus :

Le serpent. Itinéraires passés et présents. 14, 9 (2013), CNRS Éditions, 310 p. avec Cécile Davy-Rigaux et VolnyHostiou.

La musique aux expositions universelles : entre industries et cultures. 13 (2012), CNRS Éditions, 245 p. Orchestres aux XVIII et XIX siècles : composition, disposition, direction, représentation. 12 (2011), CNRS Éditions, $285 \mathrm{p}$.

\section{Choix d'articles}

"A rediscovered portrait of Henrietta-Anne of England: music, portraiture, and the arts at the Court of France ", Imago Musicae. International Yearbook of Musical Iconography XXVI (2013), p. 7-45.

« Le monument de Henry Du Mont et la sculpture funéraire à l'époque du père Ménestrier », Imago Musicae. International Yearbook of Musical Iconography XXV (2012), p. 77-106.

" The Portraits of Rameau: A Methodological Approach », Music in Art. International Journal for Music Iconography XXXVI/1-2 (2011), p. 275-300.

«Performing and Listening to Music in Paris (1770-1820): Interpreting Visual Sources », avec Michael Greenberg, Zur Aufführungspraxis von Musik der Klassik, Ute Omonsky, Hans Boje Schmuhl (ed.), Augsbourg, Wissner-Verlag, Stiftung Kloster Michaelstein, 2011, Michaelsteiner Bericht Band 76, p. 79-110.

«Les collections Henry Prunières et Geneviève Thibault de Chambure : formation, composition, interaction, valorisation ", avec Catherine Massip, Collectionner la musique : histoires d'une passion, Denis Herlin, Catherine Massip, Jean Duron, Dinko Fabris (dir.), Turnhout, Brepols, 2010, p. 217-256.

« Nouveau statut de l'instrument de musique en France au XIX ${ }^{\mathrm{e}}$ siècle dans les expositions nationales et universelles », avec Joël-Marie Fauquet. Instrumental Music and The Industrial 
Revolution, Roberto Illianoet Luca Sala (ed.), Ad Parnassum Studies 5, Bologna, Ut Orpheus Edizioni, 2010, p. 361-389.

"Curt Sachs as a Theorist for Music Museology », Music's Intellectual History, Zdravko Blazekovicet Barbara Dobbs Mackenzie (ed.), New York, Répertoire international de littérature musicale (RILM Perspectives series), 2009, p. 303-313.

"Les faiseurs d'instruments du roi ", Le prince et la musique. Les passions musicales de Louis XIV, Jean Duron (dir.), Wavre, Mardaga, 2009, p. 179-210.

« Recherche et maintien de la tradition musicale populaire en France : positions de principe, méthodes d'observation et réalisation du MNATP », Du folklore à l'ethnologie, Jacqueline Christophe, Denis-Michel Boëll, Régis Meyran (dir.), Paris, Éditions de la Maison des Sciences de l'Homme, 2009, p. 295-307.

«Considerazioni sulla conservazione degli strumenti a tastiera e linee guida per la costruzione di copie e ricostruzioni in Francia. Consideration on Keyboard Conservation and Policy for Facsimiles and Replicas in France ", Restauro e conservazione degli strumenti musicali antichi. La spinetta ovale di Bartolomeo Cristofori. Gabriele Rossi Rognoni (ed.), Florence, Nardinieditore, 2008, p. 31-52.

«Organographie et muséologie : les fondateurs d'une histoire matérielle de la musique en France ", Musica e storiaXVI/1 (2008 paru en 2011), p. 103-127.

«Philippot le Savoyard. Portraits d'un Orphée du Pont Neuf mêlés de vaudevilles, d'images et de vers burlesques ", 'L'esprit français' und die Musik Europas. Entstehung, Einflu [6: und Grenzeneinerästhetischen Doktrin. Festschrift für Herbert Schneider, Michelle Biget-Mainfroy, Rainer Schmuch (ed.), Hildesheim, Olms, 2007, p. 269-288.

«Instrument making in Lyon and Paris around 1600 », Musikinstrumentenbau-Zentrenim 16.Jahrhundert, Boje E. Hans Schmuhl, Monika Lustig (ed.), Augsbourg, Wissner-Verlag, Michaelstein, Stiftung Kloster Michaelstein, 2007, Michaelsteiner Konferenzberichte 72, p. 179-204.

« Recent Research about the Voboam Family and Their guitars », Journal of the American Musical Instrument Society XXXI (2005), p. 5-66.

\section{NOTES}

1. Jacques Thuillier (1928-2011), spécialiste de la peinture française du XVII ${ }^{\mathrm{e}}$ siècle, professeur au Collège de France à partir de 1976, titulaire de la chaire « Histoire de la création artistique en France ».

2. Georges-Henri Rivière (1897-1985), fondateur du Musée national des Arts et Traditions populaires, président du Conseil international des musées. Il a profondément marqué la muséologie contemporaine.

3. Geneviève Thibault de Chambure (1902-1975), musicologue, collectionneur et fondateur de la Société de musique d'Autrefois. Elle fut conservateur du Musée Instrumental de 1961 à 1973.

4. Musiciens des rues de Paris, catalogue d'exposition, Florence Gétreau (dir.), Paris, Éditions de la Réunion des Musées Nationaux, 1997, 142 p., 60 ill.

5. The Anthony Baines Memorial Prize de la Galpin Society for the Study of Musical Instruments (2001); le Curt Sachs Award de l'American Musical Instrument Society (2002); l'Academia Europaea (2010). 
6. Le serpent : itinéraires passés et présents. Musique $\cdot$ Images $・$ Instruments. Revue française d'organologie et d'iconographie musicale 14 (2013), CNRS Éditions, 310 p., avec Cécile Davy-Rigaux et Volny Hostiou.

7. Les cordes de l'orchestre français sous le règne de Louis XIV et Louis XV: instruments, répertoires et singularités, en collaboration avec Jean Duron. A paraître en 2014 chez Vrin.

8. Henry Prunières. Un musicologue engagé dans la vie musicale de l'entre-deux-guerres, Myriam Chimènes, Florence Gétreau, Catherine Massip (dir.), Paris, Société française de musicologie. En préparation.

9. Voir la musique, 400 p., 350 illus. À paraître chez Citadelles - Mazenod en 2014.

10. Entendre la guerre, exposition temporaire, mars-novembre 2014, Péronne, Historial de la Grande Guerre. Catalogue sous la direction de Florence Gétreau, Gallimard, 2014.

11. La facture instrumentale européenne : suprématies nationales et enrichissement mutuel, catalogue d'exposition, Paris, Société des Amis du Musée Instrumental, 1985, 248 p.

12. Instrumentistes et luthiers parisiens. XVII ${ }^{e}$ XIX ${ }^{e}$ siècles, catalogue d'exposition, Paris, Délégation à l'Action Artistique de la Ville de Paris, 1988, 254 p.

13. Musique et guerre (1914-1918), Stéphane Audoin-Rouzeau, Esteban Buch, Myriam Chimènes, Georgie Durosoir (dir.), Lyon, Symétrie, 2009.

\section{RÉSUMÉS}

Conservatrice du patrimoine pendant vingt-cinq ans, aujourd'hui directrice de recherche au CNRS habilitée à diriger des recherches, Florence Gétreau a consacré vingt années de sa carrière au Musée instrumental du Conservatoire National Supérieur de Musique de Paris et a été chef de projet du musée de la Musique (1987-1992). C'est à l'histoire des collections de ce musée que Florence Gétreau avait dédié sa thèse de doctorat, soutenue à Paris-Sorbonne en 1991.

Responsable du Département de la musique et de la parole au musée national des Arts et Traditions populaires de Paris de 1993 à 2003, elle a dirigé l'Unité de recherche associée du CNRS «Organologie et iconographie musicale» de 1992 à 1995. C'est au sein de cette équipe qu'elle a créé en 1995 la revue annuelle Musique • Images • Instruments (Klincksieck puis CNRS Éditions). Entre janvier 2004 et décembre 2013, elle dirige l'Institut de Recherche sur le Patrimoine Musical en France (UMR 200 CNRS/Culture/BnF). Depuis 1993, elle est professeur associé d'iconographie musicale et d'organologie au Conservatoire de Paris et elle a par ailleurs contribué aux enseignements du Master « Musique et musicologie » de l'Université de Tours.

Florence Gétreau nous a fait le plaisir de nous accueillir une après-midi dans son bureau à l'IRPMF, rue de Louvois, afin d'échanger avec nous sur son parcours professionnel et ses différentes manières de faire de la recherche au fil de sa riche carrière, singulière, à la croisée de différentes disciplines et de différents corps professionnels.

\section{INDEX}

Keywords : iconography, organology, museology, pluridisciplinarity, music, World War I

Mots-clés : iconographie, organologie, muséographie, pluridisciplinarité, musique, Première Guerre mondiale 


\section{AUTEURS}

\section{FANNY GRIBENSKI}

Ancienne élève de l'École Normale Supérieure de Lyon et du Conservatoire National Supérieur de Musique et de Danse de Paris, agrégée de musique, Fanny Gribenski est doctorante contractuelle à l'École des hautes études en sciences sociales (Centre Georg Simmel) où elle prépare une thèse sur L'église comme lieu de musique, Paris, 1830-1903 sous la codirection de Rémy Campos et de Patrice Veit.

\section{ISABELLE MAYAUD}

Isabelle Mayaud est doctorante à l'École des hautes études en sciences sociales (Centre Georg Simmel) et à l'Université Paris 8 (Labtop) sous la codirection de Laurent Jeanpierre et de Michael Werner. Sa thèse porte sur La production de savoirs sur les lointains musicaux en France au XIX ${ }^{e}$ siècle. 